\title{
Cholera toxin B suppresses allergic inflammation through induction of secretory $\lg \mathrm{A}$
}

\author{
HH Smits ${ }^{1,2,8}$, AK Gloudemans $^{1,8}$, M van Nimwegen $^{1}$, MA Willart $^{1,3}$, T Soullié $^{1}$, F Muskens $^{1}$, \\ EC de Jong ${ }^{4}$, L Boon ${ }^{5}$, C Pilette ${ }^{6}$, F-E Johansen ${ }^{7}$, HC Hoogsteden ${ }^{1}$, H Hammad ${ }^{1,3}$ and BN Lambrecht ${ }^{1,3}$
}

In healthy individuals, humoral immune responses to allergens consist of serum IgA and IgG4, whereas cellular immune responses are controlled by regulatory $T$ (Treg) cells. In search of new compounds that might prevent the onset of allergies by stimulating this type of immune response, we have focused on the mucosal adjuvant, cholera toxin B (CTB), as it induces the formation of Treg cells and production of IgA. Here, we have found that CTB suppresses the potential of dendritic cells to prime for Th2 responses to inhaled allergen. When we administered CTB to the airways of naïve and allergic mice, it strongly suppressed the salient features of asthma, such as airway eosinophilia, Th2 cytokine synthesis, and bronchial hyperreactivity. This beneficial effect was only transferable to other mice by transfer of $B$ but not of $T$ lymphocytes. CTB caused a transforming growth factor- $\beta$-dependent rise in antigen-specific IgA in the airway luminal secretions, which was necessary for its preventive and curative effect, as all effects of CTB were abrogated in mice lacking the luminal IgA transporting polymeric Ig receptor. Not only do these findings show a novel therapeutic avenue for allergy, they also help to explain the complex relationship between IgA levels and risk of developing allergy in humans.

\section{INTRODUCTION}

Atopy is commonly defined as a tendency of individuals to mount serum IgE responses and/or positive skin prick tests to inhaled or ingested antigens, such as house dust mite, pollen, animal dander, or peanut. The type of immunoglobulin produced by allergen-specific B cells is heavily influenced by help from T-helper 2 lymphocytes providing cognate costimulatory molecules and cytokines for immunoglobulin class switching. In allergic individuals, $\mathrm{T}$ cells proliferate in response to allergen and are biased for interleukin (IL)-4 production, thus stimulating IgE synthesis. Several studies have showed that nonallergic individuals mount a different type of immune response to allergens, as sometimes reflected by high serum levels of allergen-specific IgA and IgG4 in the absence of specific IgE. ${ }^{1,2}$ In healthy individuals, $\mathrm{T}$ cells fail to vigorously proliferate in vitro in response to allergens, ${ }^{3}$ possibly explained by the low frequency of allergen-reactive $\mathrm{T}$ cells in these individuals, or by the existence of counterbalancing regulatory $\mathrm{T}$ (Treg) cells actively suppressing responses against allergens through IL-10 and/or transforming growth factor- $\beta$ (TGF $\beta$ ). ${ }^{4,5}$ The type of immune response to inhaled allergens is influenced by several factors, including genetic makeup, timing and level of allergen exposure in early life, and exposure to exacerbating or protective environmental triggers. ${ }^{6}$ Central to the process of allergic sensitization are dendritic cells (DCs) that pick up inhaled or ingested allergens. DCs integrate many of the above factors into a signal that is sensed by T cells, and promote a stable Th2 response and a long-lasting IgE response or promote a balanced immune response free of pro-inflammatory effector cells. ${ }^{7}$

The incidence of allergic diseases, such as asthma, has risen dramatically over the last 50 years, particularly in westernized countries. ${ }^{6}$ This rise is thought to result from an imbalance in the immune system, caused by a diminished infectious pressure during childhood. New preventive strategies that might interfere with DC-driven Th2 sensitization are urgently needed to halt this epidemic. As DC function is heavily influenced by

${ }^{1}$ Department of Pulmonary Medicine, Erasmus Medical Center, Rotterdam, The Netherlands. ²Department of Parasitology, Leiden University Medical Center, Leiden, The Netherlands. ${ }^{3}$ Department of Respiratory Diseases, Laboratory of Immunoregulation and Mucosal Immunology, University of Ghent, Ghent, Belgium. ${ }^{4}$ Department of Cell Biology \& Histology, Academic Medical Center, University of Amsterdam, Amsterdam, The Netherlands. ${ }^{5}$ Bioceros B. V., Utrecht, The Netherlands. ${ }^{6}$ Laboratory of Mucosal

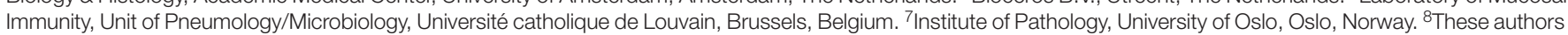
contributed equally to the work. Correspondence: BN Lambrecht (Bart.Lambrecht@UGent.be) 

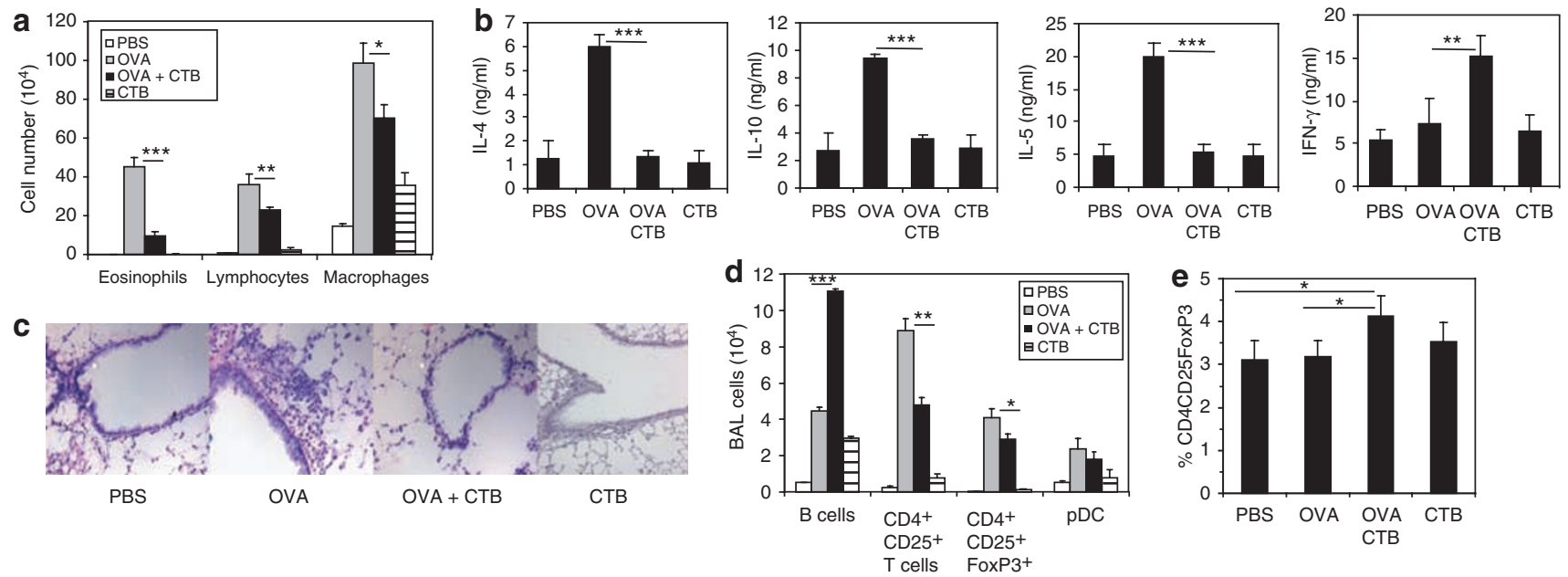

Figure 1 Cholera toxin $B(C T B)$ inhibits the potential of dendritic cells (DCs) to induce allergic sensitization. DCs were pulsed overnight by phosphatebuffered saline (PBS), ovalbumin (OVA) $(100 \mu \mathrm{g} / \mathrm{ml})$, OVA +CTB $(10 \mu \mathrm{g} / \mathrm{ml})$, or CTB $(10 \mu \mathrm{g} / \mathrm{ml})$. One million DCs were instilled in the airways of naïve mice. After 10 days, the mice were exposed to three OVA aerosols. At 1 day after the last aerosol, the mice were killed and lung lavages were taken. (a) The cellular composition of the BAL was determined by flowcytometry. (b) Mediastinal lymph node (MLN) cells were cultured with OVA (10 $\mu$ g/ml) for 4 days. Cytokine production was determined by ELISA (enzyme-linked immunosorbent assay). (c) Lungs were fixed with OCT and snap-frozen. Sections of $3-\mu \mathrm{m}$ thickness were cut and stained with hematoxylin and eosin. The number of CD4 ${ }^{+} \mathrm{CD} 25^{+}$FoxP3 $3^{+}$, pDC, and B cells in BAL (stained for CD4, CD25, FoxP3, CD19, B220, and 120G8) (d) and the number of CD4 ${ }^{+}$CD25 ${ }^{+}$FoxP3 ${ }^{+} \mathrm{T}$ cells in MLN (e) were determined by flowcytometry. Data are mean \pm s.e.m, $n=4$ to 6 mice in each group. ${ }^{\star} P<0.05,{ }^{\star *} P<0.01,{ }^{\star \star *} P<0.001$. Data from one representative experiment out of three are shown.

microbial danger signals, ${ }^{8-10}$ we searched for microbial compounds that could prevent DC-driven Th2 responses and allergic asthmatic reactions to inhaled allergens, and at the same time could directly stimulate the immune response to a type observed in healthy individuals, that is, dominated by Treg cells and $\mathrm{Ig} \mathrm{A} / \mathrm{IgG} 4$ responses.

In our search, we narrowed down on the non-toxic mucosal adjuvant cholera toxin B (CTB), produced by Vibrio Cholera. The enterotoxin cholera toxin is a holotoxin that consists of an A and B subunit, each with distinct effects on the cells of the immune system. ${ }^{11}$ The B subunit has immunomodulatory capacities, through its binding to the asialo-GM-1 receptor on $\mathrm{B}$ cells, T cells, and DCs. ${ }^{11,12}$ When CTB conjugated to allergen is administered through the oral or nasal route, it stimulates the formation of Foxp $3^{+}$IL-10-producing Treg cells, in a process requiring TGF- $\beta$ and/or IL-10. ${ }^{13-18}$ In addition, CTB is a classical mucosal adjuvant ${ }^{12,19}$ that stimulates the formation of IgA responses to ingested antigens, a process known to require TGF $\beta$ for class switching. Here, we have addressed the preventive and therapeutic potential of CTB given to the airways in a mouse model of asthma. We focused on the relative contribution of altered cellular or humoral immunity, and the importance of IL-10 and TGF $\beta$ when studying how CTB suppresses asthma. Together, our data uncovered a prominent role for mucosal IgA responses in protection from allergic disease by CTB.

\section{RESULTS \\ CTB modulates the potential of DCs to induce allergic sensitization}

To test whether CTB has the potential to interfere with DCdriven Th2 responses in the airways, we used an earlier reported model in which inflammatory-type DCs (generated in vitro by granulocyte-macrophage colony stimulating factor treatment of bone marrow precursors) were pulsed with ovalbumin (OVA) allergen and transferred into the lungs of mice, thus generating Th2 immunity and asthma features. ${ }^{20} \mathrm{In}$ mice receiving OVA-pulsed DCs, strong lymphocytosis, eosinophilia, perivascular and peribronchial inflammation, combined with goblet cell hyperplasia, were induced upon allergen challenge (Figure 1a and c). Cultures of mediastinal lymph node (MLN) cells showed the presence of Th2 cytokines, IL4, IL-5, IL-13, and IL-10 (Figure 1b). None of these were seen when phosphate-buffered saline (PBS)-pulsed DCs were injected, followed by OVA allergen challenge. The pretreatment of OVA-pulsed DCs with CTB before transfer dramatically altered their proinflammatory potential such that it prevented eosinophilic airway inflammation upon rechallenge (Figure 1a) and decreased the OVA-specific MLN Th2 cytokines (Figure 1b). In vitro CTB treatment had no toxic effects on DCs and did not alter the expression level of co-stimulatory molecules (MHCII, CD40, CD80, and CD86) known to be important for induction of T-cell responses by DCs (Supplementary Figure 1). Again, in the absence of antigen pulsing, CTB-pulsed DCs failed to induce any changes typical of allergic asthma.

Despite the increase in interferon- $\gamma$ production in MLN cultures, there were no signs of perivascular or peribronchial inflammation in the lung sections of mice receiving OVA + CTBpulsed DCs (Figure 1c). Moreover, lung mRNA analysis on several inflammatory cytokines and chemokines that are known to regulate allergic inflammation showed lower levels of IL-6, $\mathrm{MCP} 1, \mathrm{MCP} 3$, eotaxin and its receptor, CCR3, and TARC (thymus and activation regulated chemokine), indicatives of a Th2 

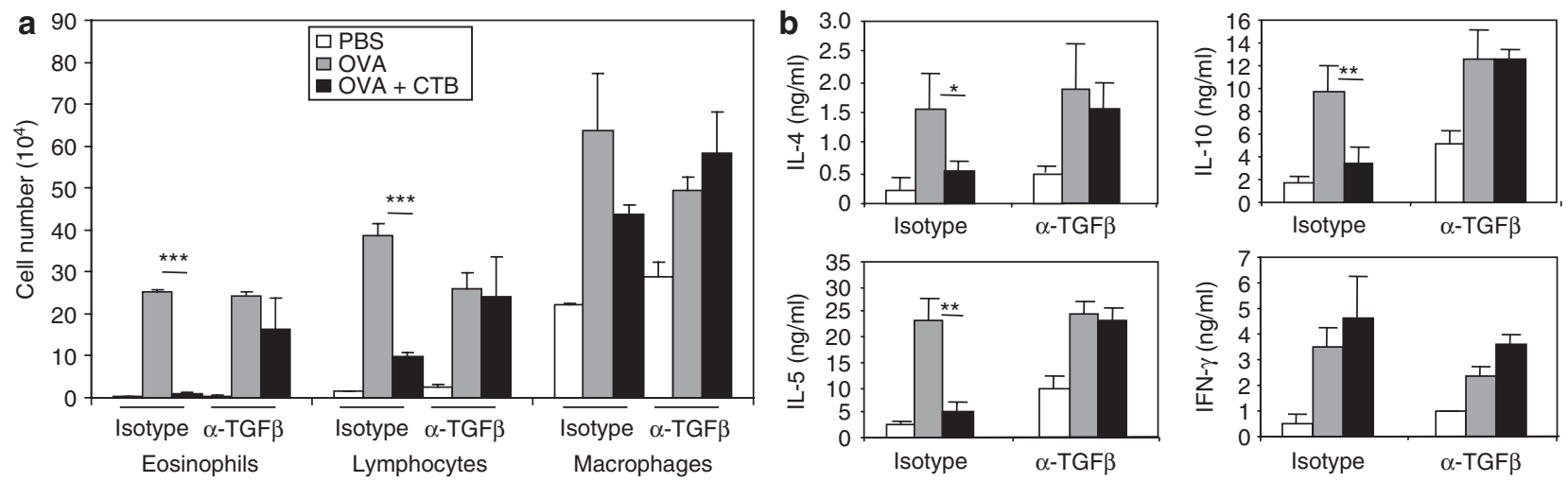

Figure 2 Involvement of TGF- $\beta$ in mediating the protective effect of cholera toxin B (CTB) on sensitization. Mice were injected with anti-tumor growth factor- $\beta$ (TGF- $\beta$ ) antibodies (11B1; $20 \mu \mathrm{g}$ ) or mouse lgG (isotype control) at day -1 till day 5 . At day 0 , mice were injected with ovalbumin (OVA)pulsed or phosphate-buffered saline (PBS)-pulsed dendritic cells, treated or not with CTB, and challenged as described in Figure 1 legend. (a) BAL cell differential counts were determined by flowcytometry. (b) Mediastinal lymph node cells were cultured with OVA (10 mg/ml) for 4 days. Cytokine production was determined by ELISA (enzyme-linked immunosorbent assay). Data are mean \pm s.e.m, $n=4$ to 6 mice in each group. ${ }^{\star} P<0.05,{ }^{\star \star} P<0.01$, ${ }^{* \star \star} P<0.001$. Data from one representative experiment out of three are shown.

signature, in mice injected with OVA + CTB-primed DCs compared with those injected with OVA-primed DCs. Importantly, IP-10 and MIG, signature of a type-1 response, were reduced after adoptive transfer of OVA + CTB-pulsed DC compared with OVA-pulsed DCs (Supplementary Figure 2), arguing against a deviation of Th2 responses toward more Th1 type of inflammation.

\section{CTB modulates the potential of DCs to induce Treg cells, yet Treg cells fail to transfer protection}

Having found that CTB treatment of DCs abolished their potential to prime for Th2 responses in the lung, we next addressed the mechanism of action. Earlier findings indicated that CTBallergen complexes given orally induced the formation of Foxp $3^{+}$ Treg cells, ${ }^{13,14}$ which had strong inhibitory effects in vitro, partly dependent on TGF- $\beta$ and IL-10. In accordance, when carrying out in vitro co-cultures of OVA + CTB-pulsed DCs together with OVA-specific $\mathrm{T}$ cells taken from $\mathrm{T}$-cell receptor transgenic mice, we observed an increased percentage of IL-10-producing $\mathrm{T}$ cells (Supplementary Figure 3a) and an elevated percentage of FoxP3 ${ }^{+}$cells (Supplementary Figure $3 \mathbf{b}$ ), when compared with OVA-pulsed DCs. However, when we transferred OVA + CTB-pulsed DCs into the airways of naïve mice in vivo, followed by OVA challenge, we found no differences in the number of $\mathrm{CD} 4{ }^{+} \mathrm{CD} 25^{\text {high }} \mathrm{FoxP}^{+} \mathrm{T}$ cells in the broncho-alveolar lavage compartment (BAL) (Figure 1d) in parallel to reduced $\mathrm{CD} 4{ }^{+} \mathrm{CD} 25^{+} \mathrm{T}$ cells and increased $\mathrm{B}$ cells-but instead small differences were found in the percentage of $\mathrm{CD} 4{ }^{+} \mathrm{CD} 25^{\text {high }} \mathrm{FoxP}^{+}$ $\mathrm{T}$ cells in the draining MLN from OVA + CTB-DC-immunized mice (Figure 1e). When MLN CD4 ${ }^{+} \mathrm{T}$ cells were taken from OVA + CTB DC-immunized mice after OVA challenge, they showed a small, yet significant capacity to reduce OVA-specific $\mathrm{T}$ cell proliferation by approximately $30 \%$ in ex vivo co-cultures of naïve $\mathrm{T}$ cells and antigen presenting cells (Supplementary Figure 4a), similar to earlier reports. ${ }^{13,14}$ This inhibitory capacity was not observed for splenic $\mathrm{CD} 4^{+} \mathrm{T}$ cells from the same mice (Supplementary Figure 4a).
Regulatory $\mathrm{T}$ cells exert their regulatory capacity through many mechanisms, including the secretion of immunoregulatory cytokines. Sorted lung $\mathrm{CD} 4^{+} \mathrm{T}$ cells taken from OVA + CTB DCimmunized mice expressed more IL-10 and TGF- $\beta$ RNA copies compared with mice that received OVA-pulsed or unpulsed DCs (Supplementary Figure 4b). Next, we addressed the contribution of Treg cell-derived immunoregulatory cytokines, IL-10 and TGF- $\beta$, during either priming dose or challenge. Administration of a neutralizing anti-IL10R antibody did not abolish the inhibitory effect of CTB treatment on allergic responses when given during priming or challenge (Supplementary Figure $5 \mathbf{a}$ and $\mathbf{b}$ ). Remarkably, TGF- $\beta$ blockade at the time of administration of OVA + CTB-pulsed DCs restored eosinophilic airway inflammation, lymphocytosis, and OVA-specific Th2 cytokines in the MLN cells (Figure $\mathbf{2 a}$ and $\mathbf{b}$ ). In contrast, blocking TGF- $\beta$ antibodies were again without effect when given during the challenge phase (Supplementary Figure 5c), arguing against the induction of TGF- $\beta$ secreting Treg cells as an explanation for allergy suppression. A classical hallmark of Treg cells is their capacity to transfer suppression when adoptively transferred to immunized recipient mice. Adoptive transfer of purified MLN T cells of OVA + CTB-DC-immunized mice into OVA-sensitized recipient mice failed, however, to influence eosinophilic airway inflammation (Supplementary Figure 5d), again arguing against a prominent role of Treg cells in mediating the protective effects of CTB in vivo.

\section{CTB stimulation of DCs suppresses allergy through induction of IgA-secreting $B$ cells}

The only consistent finding in mice receiving OVA + CTBpulsed DCs was a threefold increase in B cells in the BAL fluid, compared with mice receiving OVA-pulsed DCs (Figure 1d). This finding, together with the crucial early dependence of the protective effect of CTB on TGF- $\beta$, made us hypothesize that CTB suppressed allergy development mainly through effects on mucosal humoral immunity. TGF- $\beta$ is an important switch factor for $\mathrm{B}$ cells to produce IgA molecules ${ }^{21}$ and CTB is used as a gas- 
a

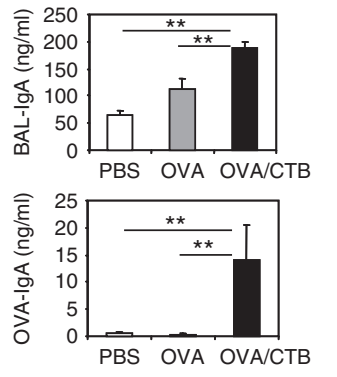

b

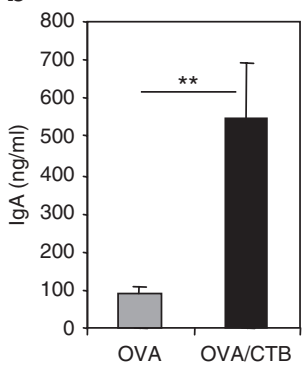

C

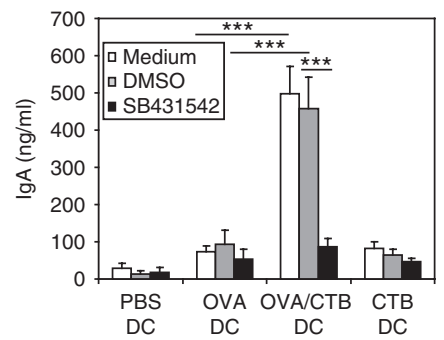

d

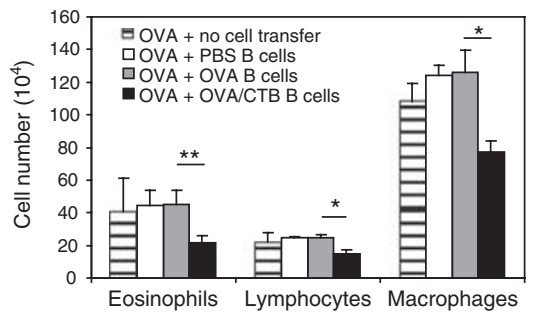

e

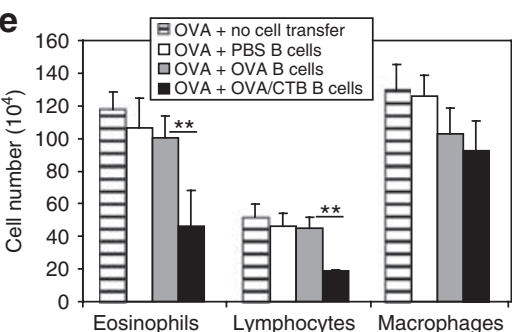

f

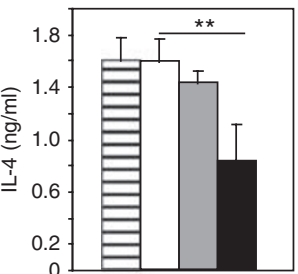

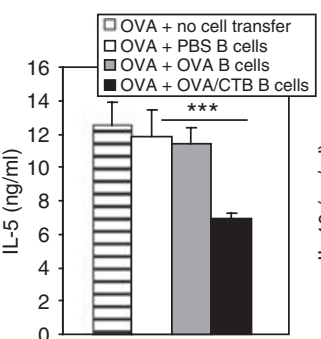
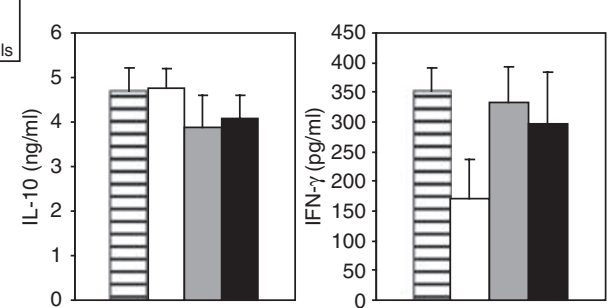

Figure 3 Contribution of IgA and B cells to the protective effect of cholera toxin B (CTB). (a) Mice were treated as described in the legend of Figure 1. Total IgA and ovalbumin (OVA)-specific IgA in BAL fluid were determined by enzyme-linked immunosorbent assay (ELISA). (b) CD19+ lung B cells were sorted. B cells were stimulated by LPS $(10 \mathrm{mg} / \mathrm{ml})$ for 7 days. Total IgA was determined by ELISA. (c) Bone marrow-derived dendritic cells (DCs) were cultured and pulsed as described in the legend of Figure 1. One million DCs were co-cultured with $1 \times 10^{6} \mathrm{CD} 19^{+}$spleen B cells, anti-lgM Fab-fragments $(10 \mu \mathrm{g} / \mathrm{ml})$, and in some conditions SB431542 $(5 \mu \mathrm{M})$ or a DMSO (dimethyl sulfoxide) control was added. After 7 days, supernatants were harvested and total IgA was measured by ELISA. (d) Mediastinal lymph node (MLN) B cells were isolated by CD19-microbeads and injected intravenously $\left(5 \times 10^{6}\right)$ in previously OVA-sensitized mice at day 10 . After two days, the mice were challenged and BAL cell differential counts were determined by flowcytometry. (e) Cells were treated as described in c. After 7 days, the B cells were harvested and injected intravenously $\left(5 \times 10^{6}\right)$ as described in $\mathbf{d}$. Mice were treated as described in d. BAL cell numbers were determined by flowcytometry. (f) MLN from the experiment described under e were taken and cells were stimulated by OVA $(10 \mathrm{mg} / \mathrm{ml})$. After 4 days, supernatants were taken and cytokines determined by ELISA. Data are mean \pm s.e.m, $n=4$ to 6 mice in each group. ${ }^{\star} P<0.05,{ }^{\star \star} P<0.01$, ${ }^{\star \star \star} P<0.001$. Data from one representative experiment out of two or three are shown.

tro-mucosal adjuvant to induce the production of IgA antibodies. ${ }^{12,19}$ To examine the possible involvement of IgA, we set out to investigate whether CTB treatment of DCs could induce local IgA production in lung mucosal tissues (Figure 3a). When OVApulsed DCs were exposed to CTB before transfer to the lungs, there was a rise in the BAL levels of total IgA and OVA-specific IgA (Figure 3a), as well as an increase in total IgA production by ex-vivo Lipopolysaccharide-stimulated CD19+ lung B cells (Figure 3b), compared with mice receiving untreated OVApulsed or PBS-pulsed DCs.

To directly address the potential of OVA + CTB-pulsed DCs to stimulate immunoglobulin class switching of B cells, we carried out in vitro co-culture of DCs with purified $\mathrm{CD} 19^{+}$spleen $\mathrm{B}$ cells, in the presence of anti-IgM Fab fragments. ${ }^{22}$ In these cultures, OVA + CTB treatment of DCs induced an increase in IgA production, an effect not seen when cells were unpulsed or pulsed with CTB alone or OVA alone (Figure 3c). Importantly, under conditions of OVA + CTB stimulation of DCs, the production of IgG1, IgG2a, or IgE was hardly induced (Supplementary Figure 6) and could not be explained by differences in B-cell proliferation as equal proliferation was induced by either OVA or OVA + CTB-pulsed DCs. Control cultures of splenic or lung B cells stimulated with CTB directly in the absence of DCs did only induce background levels of total IgA (data not shown). Although we could not detect differences in DC-TGF- $\beta$ mRNA levels between the groups, the IgA induction by OVA + CTB- pulsed DCs seemed to be dependent on TGF- $\beta$ receptor signaling, as the addition of a SMAD3 inhibitor (a TGF- $\beta$-specific signaling molecule) substantially abolished the IgA production induced by OVA + CTB-pulsed DCs (Figure 3c).

To investigate whether $\mathrm{B}$ cells were responsible for the reduction in eosinophilic airway inflammation upon allergen challenge in OVA + CTB-DCs-treated mice, MLN B cells taken from mice instilled with differently treated DCs were adoptively transferred to OVA-sensitized mice. Upon OVA challenge, eosinophilia and lymphocytosis were significantly reduced in mice injected with B cells from OVA + CTB-DCs-immunized mice, in contrast to mice injected with $B$ cells from OVA-DCsimmunized mice or mice receiving no $B$ cells (Figure 3d). The injection of naïve B cells or B cells from KLH + CTB-DCsimmunized mice was also without effect (data not shown). Similarly, adoptive transfer of in vitro-modulated $\mathrm{B}$ cells from co-cultures of OVA + CTB-pulsed DC and CD19 ${ }^{+}$spleen B cells reduced eosinophilic airway inflammation (Figure $3 \mathbf{e}$ ) and Th2 cytokine production (Figure 3f) to the same extent, as did ex vivo-isolated MLN B cells (Figure 3d).

\section{The protective effect of CTB-induced B cells on allergy depends on IgA secretion in the luminal compartments}

As it was shown earlier that IgA administered directly into the lung can capture and neutralize inhaled allergen, ${ }^{23}$ it was important to know whether the protective effect of IgA-producing B 
a

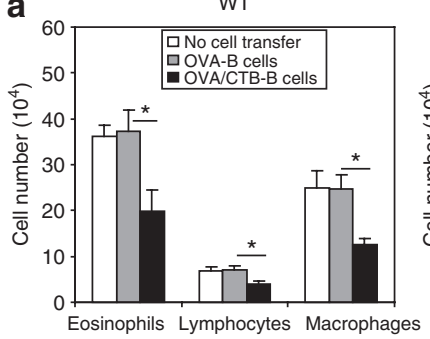

b

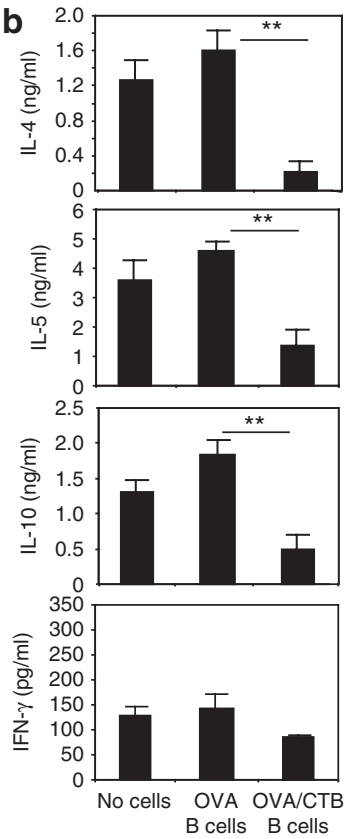

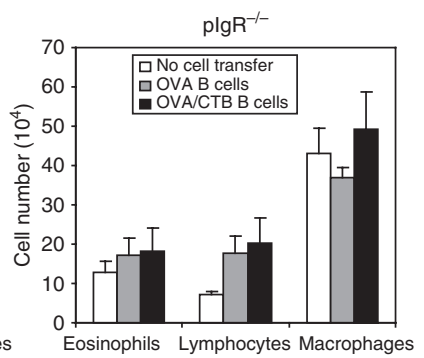
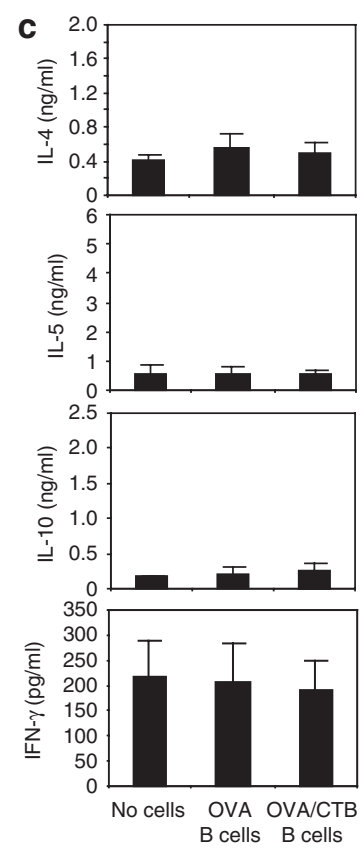

Figure 4 Cholera toxin B (CTB)-induced B cells transfer suppression of allergy by secretory IgA. Mice were injected with pulsed bone marrow-derived dendritic cells and challenged as described in Figure 1 legend. Mediastinal lymph node (MLN) B cells were isolated by CD19-microbeads and intravenously injected $\left(5 \times 10^{6}\right)$ in previously ovalbumin (OVA)-sensitized wild-type (WT) or plgR $^{-/-}$mice at day 10. After 2 days, the mice were challenged and BAL cell differential counts were determined by flowcytometry (a). MLN cells were cultured with OVA $(10 \mathrm{mg} / \mathrm{ml})$ for 4 days. Cytokine production was determined by ELISA (enzyme-linked immunosorbent assay) (b: WT; c: plgR $^{-/-}$). Data are mean \pm s.e.m, $n=4$ to 6 mice in each group. ${ }^{*} P<0.05,{ }^{* *} P<0.01$. Data from one representative experiment out of two or three are shown.

cells on allergic inflammation was because of secretory IgA being present in the airway lumen. For this, we used mice deficient for the polymeric Ig receptor (pIgR), which is necessary for transport of dimeric IgA across the epithelium into the lumen of mucosal areas. ${ }^{24}$ The B cells taken from OVA- or OVA + CTBpulsed DC-immunized mice were adoptively transferred into OVA-sensitized pIgR-deficient mice. In contrast to transfer into wild-type (WT) mice (Figure 4a), we found no reduction in eosinophilic airway inflammation, airway lymphocytosis, and MLN Th2 cytokines by adoptive transfer of B cells into pIgR-deficient mice (Figure $\mathbf{4 b}$ and $\mathbf{c}$ ). It is noteworthy that OVA sensitization by OVA-alum injections in pIgR-deficient mice resulted in much weaker eosinophilic airway inflammation and MLN Th2 cytokines (in particular IL-5) than those in WT mice.

The inhibitory effects of CTB on DC-driven Th2 development were also observed when we used CTB in vivo. In a model of sensitization to inhaled OVA through the respiratory route, we could show that intratracheal administration of CTB prevented the onset of Th2 sensitization, as a dose-dependent decrease in BAL eosinophilia, lymphocytosis, and LN Th2 cytokines was observed (Supplementary Figure $7 \mathbf{a}$ and $\mathbf{b}$ ), whereas LN interferon- $\gamma$ secretion remained unchanged. In addition, IgA production by ex vivo LPS-stimulated B cells was increased by increasing dosages of intratracheally applied CTB (Supplementary Figure 7c).

\section{Intrapulmonary administration of CTB reduces allergic airway inflammation in OVA-sensitized mice}

In view of the powerful preventive effect of CTB on development of DC-driven allergic inflammation by induction of IgA, we also wanted to obtain information whether CTB could be used in a secondary prevention setting, that is, in mice that are already sensitized to OVA. To this end, OVA-alum-sensitized mice were injected intratracheally with PBS or CTB before each OVA aerosol challenge. CTB induced a significant and dose-dependent decrease in BAL eosinophilia and lymphocytosis (Figure 5a), LN Th2 cytokines (Figure 5b), airway resistance in response to methacholine (Figure 5c), and a reduction in the BAL levels of Th2-selective chemokines (MDC and TARC; Figure 5d), than in PBS-treated mice, thus showing that CTB can also reduce Th2-mediated allergic features in sensitized mice. To address a possible role for secreted IgA in the therapeutic efficiency of CTB in already primed mice, we also applied CTB into the lungs of OVA-sensitized WT or $\mathrm{pIgR}^{-1-}$ mice before each OVA challenge, revealing a significant reduction in airway eosinophilia (Figure 5e) and LN Th2 cytokines (data not shown) in WT mice, but unchanged parameters in $\mathrm{pIgR}^{-/-}$mice.

\section{DISCUSSION}

We show here that CTB strongly suppresses the cardinal features of allergic airway inflammation when applied locally to the lung through a mechanism involving IgA-secreting protective B cells. Although we cannot exclude the indirect stimulatory effects of CTB by interaction with other cell types, such as bronchial epithelial cells, we believe that CTB has this effect by altering DC function, thus programming DCs to induce IgA-producing B cells. This conclusion is based not only on the fact that in vivo adoptive transfer of CTB-pulsed DCs leads to an induction of IgA-producing B cells but also on the fact that in vitro co-culture of bone marrow-derived DCs that were exposed to CTB with purified $\mathrm{CD} 19^{+} \mathrm{B}$ cells lead to a similar induction of IgA-producing B cells. IgA-producing B cells that were induced under both conditions protected from allergic inflammation upon transfer in OVA-sensitized recipient mice (Figure 3). Furthermore, we observed inhibitory effects of pulmonary administration of CTB on DC-driven Th2 development (Supplementary Figure 7) in a recently described model of respiratory sensitization to inhaled allergen that depends totally on the function of conventional lung DCs, as plasmacytoid DCs are depleted using depleting antibodies. ${ }^{25}$ Supporting the idea that 
a
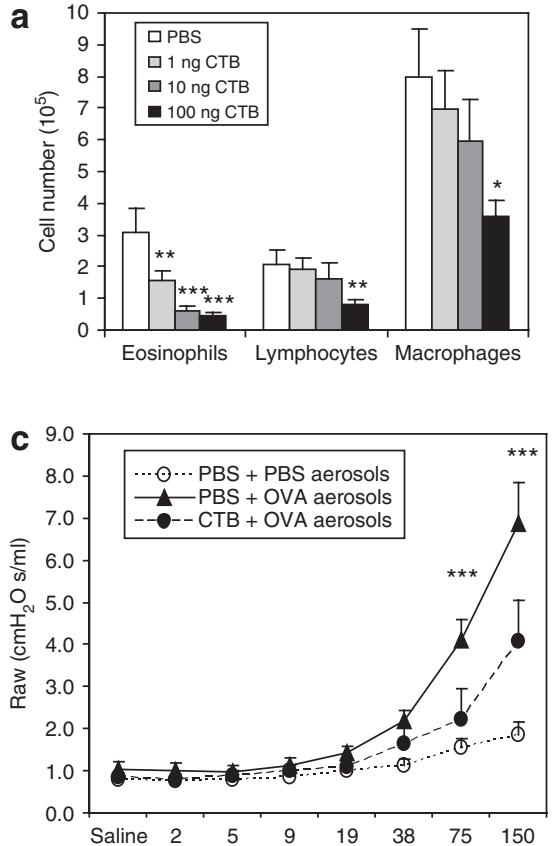
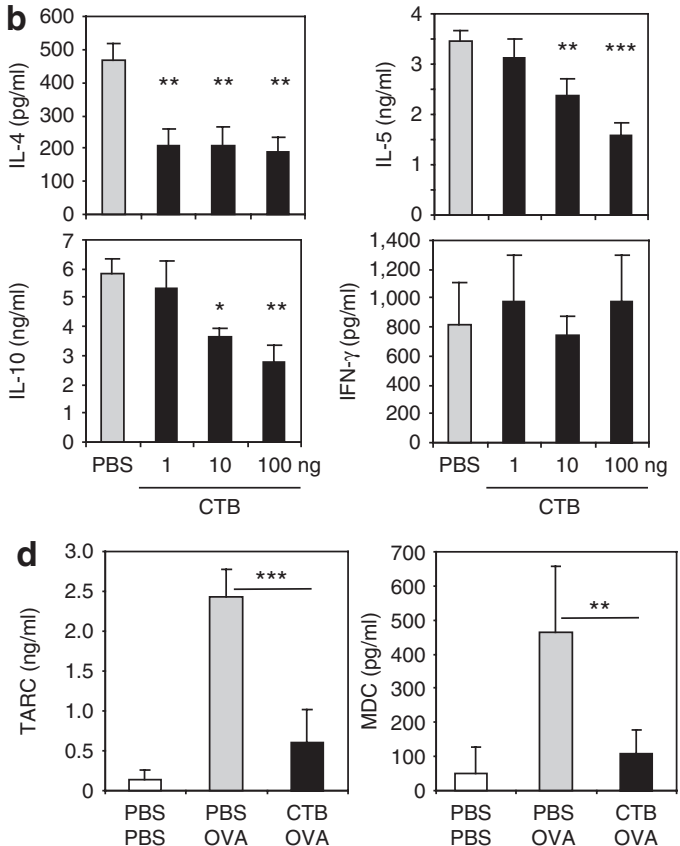
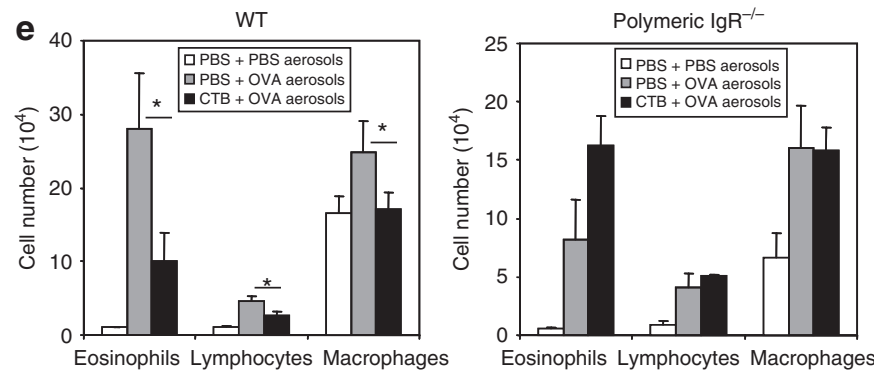

Figure 5 Intrapulmonary administration of cholera toxin B (CTB) reduces allergic airway inflammation and bronchial hyperreactivity in already sensitized mice. Mice were ovalbumin (OVA)-sensitized by two injections of OVA/alum 1 week apart. At day 17, the mice were challenged by three OVA aerosols. Before each aerosol, the mice were intratracheally instilled with phosphate-buffered saline (PBS) or CTB. (a) BAL cell differential counts were determined by flowcytometry. (b) Mediastinal lymph node cells were cultured with OVA $(10 \mathrm{mg} / \mathrm{ml})$ for 4 days. Cytokine production was determined by enzyme-linked immunosorbent assay (ELISA) (c) At 1 day after challenge, bronchial hyperreactivity was measured by invasive measurements of dynamic airway resistance (Flexivent system) in response to increasing concentrations of intravenously administered methacholine (Sigma) during mechanical ventilation. (d) Chemokine production (TARC and MDC) in BAL supernatant was determined by ELISA. (e) Wild-type and plgR ${ }^{-/-}$mice were OVA-sensitized. At day 17, the mice were PBS or OVA challenged. Before each aerosol, the mice were instilled with PBS or CTB. BAL cell differential counts were determined by flowcytometry. Data are mean \pm s.e.m, $n=6$ to 8 mice in each group. ${ }^{\star} P<0.05,{ }^{* \star} P<0.01,{ }^{\star \star \star} P<0.001$. Data from one representative experiment out of two or three are shown.

CTB can alter the function of DCs, other investigators showed that CTB induces the accumulation of DCs in the exposed epidermis and dermis, ${ }^{9}$ whereas oral feeding of CTB leads to the recruitment of CD11 $\mathrm{c}^{+} \mathrm{DC}$ in the intestinal epithelium ${ }^{10}$ and migration of subepithelial DCs into mucosal lymphoid tissues of Peyer's patches. ${ }^{26}$

Earlier reports have suggested that sublingual or oral application of CTB resulted in enhanced Treg cell development, as increased FoxP3 expression was shown in spleen, Peyer's patches, and mesenteric LN. ${ }^{13,14}$ In those studies, the Treg cells had strong inhibitory effects in vitro, that was, in part, dependent on TGF- $\beta$ or IL-10. In our study, CTB-modulated DC also primed for the development of IL-10-producing FoxP3 ${ }^{+}$Treg cells both in vitro and in vivo. However, the in vivo-generated Treg cells only displayed a mild inhibitory function in ex vivo cultures with OVA-specific T-cell receptor-transgenic T cells and antigen presenting cells (Supplementary Figure 4). The differences in Treg-cell development observed between the various studies that have used CTB as an adjuvant for immunotherapy might be explained by differences in the route of application, the nature of the allergen, or its covalent binding to CTB, as already suggested by Wiedermann et al. ${ }^{16}$

Secretory IgA is a crucial component of first-line immune mechanisms at mucosal surfaces and has many antiinflammatory functions that might suppress allergy. ${ }^{27-29}$ Supporting evidence for a regulatory role of IgA against allergic diseases comes from patients with selective IgA deficiency, who are at increased risk for auto-immune and allergic disorders. ${ }^{30}$ Furthermore, antigen-specific IgA is detected in higher levels in BAL from healthy individuals than from allergic subjects ${ }^{31}$ and 
in serum from tolerized allergic patients following successful allergen immunotherapy. ${ }^{32,33}$ Our data suggest that intrapulmonary administration of CTB can prevent the onset of allergic inflammation predominantly by inducing this secretory IgA response. The CTB or CTB-induced protective $\mathrm{B}$ cells clearly do not affect eosinophilic airway inflammation in $\mathrm{pIgR}^{-/-}$mice, despite the fact that allergic airway inflammation in OVAsensitized $\mathrm{pIgR}^{-1-}$ mice was much lower than that for WT mice. Future studies will have to address in greater detail the reason for this reduced allergic lung inflammation in $\mathrm{pIgR}^{-/-}$mice subjected to a classical asthma protocol, although the extremely high polymeric (but non-secretory) IgA levels might be involved in altering the immune responsiveness of these mice to the asthma protocol. ${ }^{24}$

One of the mechanisms by which secretory IgA could reduce allergic airway inflammation includes the capturing and neutralization of the allergen, in this way avoiding IgE-mediated effector responses. Nevertheless, intranasal treatment of mice with antigen-specific monoclonal IgA antibody also resulted in increased IgG2a serum concentrations, suggesting the influence of IgA on systemic immune responses, beyond the point of molecular allergen avoidance. ${ }^{23}$ Indeed, recent studies have shown that FcaRI (CD89) can act as an inhibitory receptor in humans. Serum IgA or anti-FcaRI Fab inhibits activating responses of heterologous $\mathrm{Fc} \gamma \mathrm{R}$ or the high-affinity IgE receptor, FceRI. ${ }^{34}$ Most strikingly, anti-Fc $\alpha$ RI Fab treatment suppressed allergic asthma manifestations in humanized Fc $\alpha$ RI-transgenic mice. ${ }^{34}$ Unfortunately, no mouse counterpart or alternative IgA receptors for the human CD89 have been identified yet.

One aspect of our study that clearly stands out is the fact that CTB programs the bone marrow DCs to instruct B cells for IgA class switching. Earlier, it was reported that B cells class switch to IgA production only when exposed to mucosal DC, such as Peyer's patch DCs or lung DCs, ${ }^{22}$ switching being explained through the production of mucosal-specific specializations of DCs, such as production of retinoic acid and/or TGF- $\beta$. As our data show that CTB exposure of bone marrow DCs endows them with the capacity to promote IgA class switching, it will be very interesting to study whether CTB also induces instructive signals normally associated with mucosal Peyer's patch DCs. At least the signaling through the TGF $\beta$ receptor on $B$ cells was necessary to induce IgA production in vitro, as revealed by the use of a SMAD3 inhibitor. Future studies will have to address the functional importance of retinoic acid metabolites in this process.

In conclusion, we show that CTB is endowed with the potential to inhibit Th2 sensitization while at the same time stimulating features of the response of non-allergic individuals to allergens, one being the induction of Treg cells ${ }^{13,14}$ and the other being the induction of an immunoglobulin response dominated by IgA. Owing to the absence of a correlate for human IgG4 in the mouse, we could not address any effects that CTB might have on IgG4. In search for alternative therapies for allergic diseases, CTB forms a highly promising lead molecule as it uniquely induces protection against allergic airway inflammation through a novel mechanism targeting DC function and resulting in protective IgA.

\section{METHODS}

\section{Animals}

Female BALB/c mice (6-week-old) were purchased at Harlan (Horst, The Netherlands) and kept under specific pathogen-free conditions according to the institutional guidelines of the animal ethics and welfare committee. $\mathrm{PIgR}^{-1-}$ mice were provided by Dr Johansen (Oslo, Norway).

\section{A mouse model of asthma induced by adoptive transfer of bone marrow-derived DC}

The DCs were generated by culturing bone marrow cells in a medium containing recombinant granulocyte-macrophage colony stimulating factor, as described. ${ }^{20}$ After 7 days, the cells were pulsed overnight with PBS, OVA $(100 \mu \mathrm{g} / \mathrm{ml}$, Worthington Biochemical Corp., Lakewood, NJ), or OVA + CTB (10 ig/ml, Sigma-Aldrich, St Louis, MO). DCs $\left(1 \times 10^{6}\right)$ were instilled through the opening vocal cords, as described. ${ }^{20}$ On days $10-12$, the mice were exposed to OVA aerosols and killed $24 \mathrm{~h}$ after the last aerosol. BAL was carried out and lungs and MLNs were removed and digested using collagenase/DNAse as described.$^{35}$ In some experiments, TGF- $\beta$-Ab $(50 \mu \mathrm{g}$, clone 1D11, obtained from ATCC, Manassas, VA) or mouse IgG (isotype control) was daily administered intraperitoneally (IP) at day - 1 till day 5 (before and during priming) or from day 10 till day 13 (before and during challenge), or IL-10R-Ab $(250 \mu$; clone 1B1.3a, obtained from Dr K Moore (DNAX, Palo Alto, CA)) or rat IgG1 (isotype control) was administered IP at day -1 (before priming) or day 10 (before challenge).

\section{A mouse model of asthma-induced systemic IP sensitization} Mice were sensitized by two IP injections of OVA $(10 \mu \mathrm{g})$ emulsified in $\mathrm{AL}(\mathrm{OH})_{3}(1 \mathrm{mg}$; Imject Alum, Pierce, Rockford, IL) on day 0 and day 7. At days 17-19, mice were challenged with either PBS or OVA aerosols (grade III, $10 \mathrm{mg} / \mathrm{ml}$ in PBS; $30 \mathrm{~min}$, Sigma). In some experiments, mice were isofluorane-anesthetized $30 \mathrm{~min}$ before each aerosol and received an intratracheal injection of PBS or purified CTB (Sigma). At $24 \mathrm{~h}$ after the last exposure, BAL was carried out, and lungs and MLNs were removed, as described. ${ }^{35}$

\section{Airway hyperresponsiveness}

Airway hyperreactivity was assessed by invasive measurements of dynamic airway resistance in response to increasing concentrations of intravenously administered metacholine (Sigma) during mechanical ventilation with a Flexivent apparatus (SCIREQ, Montreal, QC, Canada), as described earlier. ${ }^{36}$

\section{Adoptive transfer of MLN cells}

At day 17, OVA-sensitized mice (two IP injections of OVA/alum; day 0 and day 7) received intravenously $5 \times 10^{6} \mathrm{MLN} \mathrm{CD} 19^{+} \mathrm{B}$ cells (positive isolation by CD19+ MACS microbeads; Miltenyi, Bergisch Gladbach, Germany) or $5 \times 10^{6}$ MLN CD4 ${ }^{+}$T cells (positive MACS isolation kit for $\mathrm{CD}^{+} \mathrm{T}$ cells; Miltenyi) from donor mice treated as indicated in the legends and challenged with OVA aerosols. After 2 days, the recipient mice were challenged with daily OVA aerosols for 3 consecutive days.

\section{Flowcytometry and sorting}

BAL cells were stained with antibodies directed against MHCII, CD11c, CD3, CD19 (all from eBioScience, SanDiego, CA), and CCR3 (R\&D systems, Abingdon, UK), as described before. ${ }^{37}$ For isolation of $B$ cells from lung suspensions, B cells were pre-purified using CD19+ $\mathrm{MACS}$ microbeads (Miltenyi) and subsequently sorted on B220 and CD19 positivity using a FacsARIA flowcytometer (BD Bioscience, Franklin Lakes, NJ).

\section{Culture of $B$ cells and IgA measurements}

The CD $19^{+}$lung B cells $\left(2 \times 10^{5}\right.$ cells $)$ were stimulated with $10 \mu \mathrm{g} / \mathrm{ml}$ LPS (Sigma). After 7 days, the supernatant was harvested and total IgA or OVA-specific IgA was measured by ELISA (BD Bioscience, Franklin Lakes, NJ) (enzyme-linked immunosorbent assay) (BD). 
Differentially pulsed or unpulsed DCs were co-cultured with MACSsorted splenic $\mathrm{CD} 19^{+} \mathrm{B}$ cells $\left(1 \times 10^{6}\right.$ cells per cell type in a ratio $\left.1: 1\right)$ and anti-mouse IgM F $\left(\mathrm{ab}^{\prime}\right)_{2}(10 \mu \mathrm{g} / \mathrm{ml}$; Jackson Immunoresearch Laboratories, West Grove, PA). In some conditions, SB431542, a SMAD3 inhibitor ( $5 \mu \mathrm{M}$; Sigma-Aldrich) or a DMSO (dimethyl sulfoxide) control was added. After 7 days, the supernatant was harvested and total IgA, IgG1, IgG2a, or IgE were measured by ELISA (BD). The detection limit was $2 \mathrm{ng} / \mathrm{ml}$.

\section{Cytokine measurements}

Cells were plated at a density of $2 \times 10^{5}$ cells per well and restimulated with $10 \mu \mathrm{g} / \mathrm{ml}$ OVA. After 4 days, IL-4, IL-5, IL-10, interferon- $\gamma$ (OptEIA, Pharmingen, Becton Dickinson, San Diego, CA) and IL-13 (R\&D systems) were measured in supernatants by ELISA. The detection limit of the ELISA-kits is generally $5-10 \mathrm{pg} / \mathrm{ml}$.

\section{Statistical analysis}

For statistical analysis, Kruskal-Wallis one-way ANOVA (analysis of variance) was used to calculate the overall $P$-values between the groups. In case of a significant difference in the Kruskal-Wallis analysis, the Mann-Whitney $U$ test was used for unpaired, non-parametric data to compare the individual groups separately. $P$-values $<0.05$ were considered significant. $P$-values less than $0.05,0.01$, or 0.001 are indicated by one, two, or three asterisks.

SUPPLEMENTARY MATERIAL is linked to the online version of the paper at http://www.nature.com/mi

\section{ACKNOWLEDGMENTS}

This work was supported by VENI grants (to H.S. and H.H.), ZonMW (to B.L.) and VIDI grant (to B.L) of the Dutch Organization of Science, a grant from the Netherlands Asthma Foundation (to A.G.) and an Odysseus grant of the Flemish Government (to B.L.).

\section{DISCLOSURE}

The authors declare no conflict of interest.

2009 Society for Mucosal Immunology

\section{REFERENCES}

1. Jutel, M., Akdis, M., Budak, F., Aebischer-Casaulta, C., Wrzyszcz, M. \& Blaser, K. et al. IL-10 and TGF-beta cooperate in the regulatory $T$ cell response to mucosal allergens in normal immunity and specific immunotherapy. Eur. J. Immunol. 33, 1205-1214 (2003).

2. Aydogan, M., Mete, N., Yazi, D., Akkoc, T., Ozdemir, C. \& Blaser, K. et al. Comparison of Der p1-specific antibody levels in children with allergic airway disease and healthy controls. Pediatr. Allergy Immunol. 18, 320-325 (2007).

3. Wierenga, E.A., Snoek, M., Bos, J.D., Jansen, H.M. \& Kapsenberg, M.L. Comparison of diversity and function of house dust mite-specific $T$ lymphocyte clones from atopic and non-atopic donors. Eur. J. Immunol. 20, 1519-1526 (1990).

4. Ling, E.M., Smith, T., Nguyen, X.D., Pridgeon, C., Dallman, M. \& Arbery, J. et al. Relation of CD4+CD25+ regulatory T-cell suppression of allergen-driven T-cell activation to atopic status and expression of allergic disease. Lancet 363, 608-615 (2004).

5. Akdis, C.A., Blaser, K. \& Akdis, M. Genes of tolerance. Allergy 59, 897-913 (2004).

6. Umetsu, D.T., McIntire, J.J., Akbari, O., Macaubas, C. \& DeKruyff, R.H. Asthma: an epidemic of dysregulated immunity. Nat. Immunol. 3, 715-720 (2002).

7. Hammad, H. \& Lambrecht, B.N. Dendritic cells and epithelial cells: linking innate and adaptive immunity in asthma. Nat. Rev. Immunol. 8, 193-204 (2008).

8. Reis e Sousa Diebold, S.D., Edwards, A.D., Rogers, N., Schulz, O. \& Sporri, R. Regulation of dendritic cell function by microbial stimuli. Pathol. Biol. (Paris) 51, 67-68 (2003).

9. Anjuere, F., George-Chandy, A., Audant, F., Rousseau, D., Holmgren, J. \& Czerkinsky, C. Transcutaneous immunization with cholera toxin B subunit adjuvant suppresses IgE antibody responses via selective induction of Th1 immune responses. J. Immunol. 170, 1586-1592 (2003).

10. Anjuere, F., Luci, C., Lebens, M., Rousseau, D., Hervouet, C. \& Milon, G. et al. In vivo adjuvant-induced mobilization and maturation of gut dendritic cells after oral administration of cholera toxin. J. Immunol. 173, 5103-5111 (2004).

11. Lycke, N. From toxin to adjuvant: basic mechanisms for the control of mucosal IgA immunity and tolerance. Immunol. Lett. 97, 193-198 (2005).

12. Holmgren, J. \& Czerkinsky, C. Mucosal immunity and vaccines. Nat. Med. 11, S45-S53 (2005).

13. Sun, J.B., Cuburu, N., Blomquist, M., Li, B.L., Czerkinsky, C. \& Holmgren, $J$ S Sublingual tolerance induction with antigen conjugated to cholera toxin B subunit induces Foxp3+CD25+CD4+ regulatory T cells and suppresses delayed-type hypersensitivity reactions. Scand. J. Immunol. 64, 251-259 (2006).

14. Sun, J.B., Raghavan, S., Sjoling, A., Lundin, S. \& Holmgren, J. Oral tolerance induction with antigen conjugated to cholera toxin B subunit generates both Foxp3+CD25+ and Foxp3-. J. Immunol. 177, 7634-7644 (2006).

15. Sun, J.B., Holmgren, J. \& Czerkinsky, C. Cholera toxin B subunit: an efficient transmucosal carrier-delivery system for induction of peripheral immunological tolerance. Proc. Natl. Acad. Sci. USA 91, 10795-10799 (1994).

16. Wiedermann, U., Jahn-Schmid, B., Lindblad, M., Rask, C., Holmgren, J. \& Kraft, D. et al. Suppressive versus stimulatory effects of allergen/ cholera toxoid (CTB) conjugates depending on the nature of the allergen in a murine model of type I allergy. Int. Immunol. 11, 1717-1724 (1999).

17. Bublin, M., Hoflehner, E., Wagner, B., Radauer, C., Wagner, S. \& Hufnagl, $\mathrm{K}$. et al. Use of a genetic cholera toxin B subunit/allergen fusion molecule as mucosal delivery system with immunosuppressive activity against Th2 immune responses. Vaccine 25, 8395-8404 (2007).

18. Lavelle, E.C., McNeela, E., Armstrong, M.E., Leavy, O., Higgins, S.C. \& Mills, K.H. Cholera toxin promotes the induction of regulatory T cells specific for bystander antigens by modulating dendritic cell activation. J. Immunol. 171, 2384-2392 (2003).

19. Holmgren, J., Adamsson, J., Anjuère, F., Clemens, J., Czerkinsky, C. \& Eriksson, K. et al. Mucosal adjuvants and anti-infection and antiimmunopathology vaccines based on cholera toxin, cholera toxin B subunit and CpG DNA. Immunol. Lett. 97, 181-188 (2005).

20. Lambrecht, B.N., De Veerman, M., Coyle, A.J., Gutierrez-Ramos, J.C., Thielemans, K. \& Pauwels, R.A. Myeloid dendritic cells induce Th2 responses to inhaled antigen, leading to eosinophilic airway inflammation. J. Clin. Invest. 106, 551-559 (2000).

21. Kim, P.H., Eckmann, L., Lee, W.J., Han, W. \& Kagnoff, M.F. Cholera toxin and cholera toxin B subunit induce lgA switching through the action of TGF-beta 1. J. Immunol. 160, 1198-1203 (1998).

22. Mora, J.R., Iwata, M., Eksteen, B., Song, S.Y., Junt, T. \& Senman, B. et al. Generation of gut-homing IgA-secreting $B$ cells by intestinal dendritic cells. Science 314, 1157-1160 (2006)

23. Schwarze, J., Cieslewicz, G., Joetham, A., Sun, L.K., Sun, W.N. \& Chang, T.W. et al. Antigen-specific immunoglobulin-A prevents increased airway responsiveness and lung eosinophilia after airway challenge in sensitized mice. Am. J. Respir. Crit. Care Med. 158, 519-525 (1998).

24. Johansen, F.E., Pekna, M., Norderhaug, I.N., Haneberg, B., Hietala, M.A. \& Krajci, P. et al. Absence of epithelial immunoglobulin A transport, with increased mucosal leakiness, in polymeric immunoglobulin receptor/ secretory component-deficient mice. J. Exp. Med. 190, 915-922 (1999).

25. de Heer, H.J., Hammad, H., Soullié, T., Hijdra, D., Vos, N. \& Willart, M.A. et al. Essential role of lung plasmacytoid dendritic cells in preventing asthmatic reactions to harmless inhaled antigen. J. Exp. Med. 200, 89-98 (2004).

26. Anosova, N.G., Chabot, S., Shreedhar, V., Borawski, J.A., Dickinson, B.L. \& Neutra, M.R. Cholera toxin, E. coli heat-labile toxin, non-toxic derivatives induce dendritic cell migration into the follicle-associated epithelium of Peyer's patches. Mucosal Immunol. 1, 59-67 (2008).

27. Kerr, M.A. The structure and function of human IgA. Biochem. J. 271, 285-296 (1990).

28. Corthesy, B., Gaskins, H.R. \& Mercenier, A. Cross-talk between probiotic bacteria and the host immune system. J. Nutr. 137, 781S-790S (2007).

29. Pilette, C., Durham, S.R., Vaerman, J.P. \& Sibille, Y. Mucosal immunity in asthma and chronic obstructive pulmonary disease: a role for immunoglobulin A? Proc. Am. Thorac. Soc. 1, 125-135 (2004). 
30. Schaffer, F.M., Monteiro, R.C., Volanakis, J.E. \& Cooper, M.D. IgA deficiency. Immunodefic. Rev. 3, 15-44 (1991).

31. Peebles, R.S. Jr, Liu, M.C., Adkinson, N.F. Jr, Lichtenstein, L.M. \& Hamilton, R.G. Ragweed-specific antibodies in bronchoalveolar lavage fluids and serum before and after segmental lung challenge: IgE and IgA associated with eosinophil degranulation. J. Allergy Clin. Immunol. 101, 265-273 (1998).

32. Pilette, C., Nouri-Aria, K.T., Jacobson, M.R., Wilcock, L.K., Detry, B. \& Walker, S.M. et al. Grass pollen immunotherapy induces an allergenspecific IgA2 antibody response associated with mucosal TGF-beta expression. J. Immunol. 178, 4658-4666 (2007).

33. Sletten, G.B., Halvorsen, R., Egaas, E. \& Halstensen, T.S. Casein-specific immunoglobulins in cow's milk allergic patient subgroups reveal a shift to IgA dominance in tolerant patients. Pediatr. Allergy Immunol. 18, 71-80 (2007).
34. Pasquier, B., Launay, P., Kanamaru, Y., Moura, I.C., Pfirsch, S. \& Ruffié, C. et al. Identification of FcalphaRI as an inhibitory receptor that controls inflammation: dual role of FcRgamma ITAM. Immunity 22, 31-42 (2005).

35. van Riit, L.S., Jung, S., Kleinjan, A., Vos, N., Willart, M. \& Duez, C. et al. In vivo depletion of lung CD11C+ dendritic cells during allergen challenge abrogates the characteristic features of asthma. J. Exp. Med. 201, 981-991 (2005).

36. Idzko, M., Hammad, H., van, Nimwegen M., Kool, M., Müller, T. \& Soullié, T. et al. Local application of FTY720 to the lung abrogates experimental asthma by altering dendritic cell function. J. Clin. Invest. 116, 2935-2944 (2006).

37. van Rijt, L.S., Kuipers, H., Vos, N., Hijdra, D., Hoogsteden, H.C. \& Lambrecht, B.N. A rapid flow cytometric method for determining the cellular composition of bronchoalveolar lavage fluid cells in mouse models of asthma. J. Immunol. Methods 288, 111-121 (2004). 